\title{
Anomaly cancellation condition in an effective nonperturbative electroweak theory
}

\author{
Vieri Mastropietro* \\ Dipartimento di Matematica, Università di Milano, Via Saldini, 50, I-20133 Milano, Italy
}

(Received 22 October 2020; accepted 7 January 2021; published 29 January 2021)

\begin{abstract}
We establish the nonperturbative validity of the gauge anomaly cancellation condition in an effective electroweak theory of massless fermions with finite momentum cutoff and Fermi interaction. The requirement that the current is conserved up to terms smaller than the energy divided by the cutoff scale, which is the natural condition as gauge invariance is only emerging, produces the same constraint on charges as in the Standard Model. The result holds at a nonperturbative level as the functional integrals are expressed by convergent power series expansions and are analytic in a finite domain.
\end{abstract}

DOI: 10.1103/PhysRevD.103.013009

\section{INTRODUCTION}

In a chiral theory, classical gauge invariance can be broken at the quantum level by anomalies, a fact producing a lack of renormalizability and of internal consistency. Therefore, in the Standard Model, the anomalies need to cancel out, and this produces an algebraic condition on the hypercharges (and, therefore, on the charges); see, e.g., [1,2], which gives a partial explanation to charge quantization without reference to grand unification. The values of hypercharges, that at a classical level can take any value, are constrained by a purely quantum effect.

In order to see how this condition arises, the gauge fields can be decomposed in classical background and quantum parts; see, e.g., [3]. Neglecting the effect of the quantum gauge fields (but keeping quantum fermions), the conservation of current, following classically by the Noether theorem, is broken by terms quadratic in the fields proportional to the three current correlations. They are expressed by the sum of a small number of terms ("triangle graphs"), and the condition on the charges comes from the request that such a sum is vanishing. When the effect of quantum gauge fields is taken into account, the anomaly is instead the sum of infinitely many graphs. Radiative corrections could produce extra conditions, but the Adler-Bardeen theorem [4] is invoked to say that this is not the case. Such a property, which says that the anomaly is not renormalized by interactions, is a perturbative statement relying on cancellations valid assuming exact symmetries and removed

\footnotetext{
*vieri.mastropietro@unimi.it
}

Published by the American Physical Society under the terms of the Creative Commons Attribution 4.0 International license. Further distribution of this work must maintain attribution to the author(s) and the published article's title, journal citation, and DOI. Funded by SCOAP. cutoff. Nonperturbative versions of such a property in a functional integral framework using the Jacobian method [5] are indeed valid only at one loop; see, e.g., [6,7].

Natural questions are if the cancellation condition is valid at a nonperturbative level and if it still holds in an effective description when some symmetry is only approximate. The two questions are related; we can consider a theory with a finite cutoff which can be possibly studied nonperturbatively, but some symmetry is necessarily broken. Such problems have been extensively analyzed over the years. Lattice gauge theory is a natural framework for a nonperturbative construction, but the program of getting an anomaly-free formulation of electroweak theory is still incomplete; see, for instance, [8-12]. In Refs. [13,14], the anomaly in a theory with a finite momentum cutoff has been considered at a perturbative level. The role of symmetry-breaking terms in the anomaly cancellation was considered in Ref. [15], finding that at one loop they do not break the cancellation condition.

In this paper, we follow a different point of view. We keep a finite momentum ultraviolet cutoff, and we still decompose the gauge field into a classical background and a quantum field; this second field is integrated out to produce an effective fermionic interaction. We consider, therefore, an effective electroweak theory of massless fermions with a finite momentum cutoff and quartic Fermi interaction. It is not indeed restrictive to consider only a quartic interaction, as, after the first renormalization group (RG) integration step, monomials of all orders are generated in the interaction. One can even look to such a theory as the generic result of integrating out high-energy degrees of freedom from some more fundamental theory; see, e.g., [16-18]. We prove that the fermionic functional integrals defining such a theory are mathematically well defined and the correlations are analytic in a finite domain of couplings of the order of the cutoff divided by the gauge 
boson masses. Analyticity follows from determinant bounds for fermionic expectations. The results hold in the limit of a removed infrared cutoff, so that the functional integrals are infinite dimensional.

The Ward identities in the effective theory have extra terms which would be formally vanishing, removing the cutoff. Keeping only classical gauge fields not taking into account interactions, the quadratic response is again the sum of regularized triangle graphs which cancel out under the cancellation condition up to terms of the order of the energy-cutoff ratio. The nonperfect cancellation is expected, as a finite momentum cutoff breaks gauge invariance already in the classical action. When the interaction is taken into account, the response is the sum of infinitely many graphs and the Adler-Bardeen argument does not hold, as it relies on symmetries which are broken by the cutoff. However, by our exact RG analysis, we show that the response can be decomposed into two terms; one is the sum of triangle graphs with dressed vertices and propagators, which cancel out under the cancellation condition, and the other is given by a complicate series of renormalized graphs which again can be rigorously bounded by a power of the energy-cutoff ratio. In conclusion, the requirement that the current is conserved up to terms of the energy divided by the cutoff scale, which is the natural condition in the effective quantum field theory as gauge symmetry is emerging, provides the same constraint on charges as is found in the Standard Model at a perturbative level. It is, therefore, a robust condition holding at a nonperturbative level in an effective theory and even when gauge symmetry is classically not exact.

The results are obtained by constructive RG methods (see, e.g., [19] for an introduction), which have been previously used to construct chiral interacting theories and the chiral anomaly in $d=1+1$ [20-22] in the limit of the removed ultraviolet cutoff. In the present case of effective electroweak in $d=3+1$, analyticity is found only with a finite cutoff of the order of the gauge mass and removed infrared cutoff, as Fermi interaction is not renormalizable.

The rest of the paper is organized in the following way. In Sec. II, we present the effective model, and we state our main result. In Sec. III, we present the renormalization group analysis, and the Appendixes contain the more mathematical details.

\section{EFFECTIVE ELECTROWEAK THEORY}

\section{A. Grassmann integration and currents}

We consider a single family of particles with two leptons $(\nu, e)$ and two quarks $(u, d)$; the quarks have another color index which takes three values. We introduce, therefore, Grassmann variables $\psi_{i, L, x}^{ \pm}$and $\psi_{i, R, x}^{ \pm}$with $L$ and $R$ denoting chirality, $x \in[-L / 2, L / 2]^{4}$ with antiperiodic boundary conditions, and $\left\{\psi_{i, s, x}^{+}, \psi_{i^{\prime}, s^{\prime}, x^{\prime}}^{+}\right\}=\left\{\psi_{i, s, x}^{+}, \psi_{i^{\prime}, s^{\prime}, x^{\prime}}^{-}\right\}=\left\{\psi_{i, s, x}^{-}, \psi_{i^{\prime}, s^{\prime}, x^{\prime}}^{-}\right\}=0$

with $s=L, R$. One introduces the doublets $\Psi_{l, x}^{ \pm}=$ $\left(\psi_{\nu, L, x}^{ \pm}, \psi_{e, L, x}^{ \pm}\right)$and $\Psi_{q, x}^{ \pm}=\left(\psi_{u, L, x}^{ \pm}, \psi_{d, L, x}^{ \pm}\right)$.

The index labeling the two components of $\psi_{x, i, s}^{ \pm}$and the color index for $\psi_{u, s, x}^{ \pm}$and $\psi_{d, s, x}^{ \pm}$are omitted.

We define $\psi_{i, s, x}^{ \pm}=\frac{1}{L^{4}} \sum_{k} e^{i k x} \hat{\psi}_{i, s, k}^{ \pm}$with $k=\frac{2 \pi}{L} n$, with $\hat{\psi}_{k, i, s}^{ \pm}$another set of Grassmann variables. We introduce a smooth momentum cutoff $\chi_{N}(k)$, which is a infinitely differentiable compact support function (this is useful to get good decay properties in coordinate space) such that $\chi_{N}(k)=0$ for $|k| \geq \gamma^{N+1}$ and $\chi_{N}(k)=1$ for $|k| \leq \gamma^{N}, \gamma>1$ a scaling parameter. Therefore, $\gamma^{N}$ is the ultraviolet cutoff, while the infrared cutoff is provided by $L$. The "fermionic Gaussian measure" is defined as $(i=\nu, e, u, d$ and $s=L, R$ )

$P(d \psi)=\left[\prod_{i, s, k}^{*} d \hat{\psi}_{i, s, k}^{+} d \hat{\psi}_{i, s, k}\right] e^{-\left(1 / L^{4}\right) \sum_{k}^{*} \hat{\psi}_{i, s, k}^{+} \chi_{N}^{-1}(k)\left(i \sigma_{\mu}^{i} k_{\mu}\right) \hat{\psi}_{i, s, k}^{-},}$

where $\prod_{k}^{*}$ is a product over $k$ in the support of $\chi_{N}(k)$ and $\sigma_{\mu}^{L}=\left(\sigma_{0}, i \vec{\sigma}\right)$ and $\sigma_{\mu}^{R}=\left(\sigma_{0},-i \vec{\sigma}\right), \vec{\sigma}=\sigma_{1}, \sigma_{2}, \sigma_{3}$, with

$\sigma_{1}=\left(\begin{array}{ll}0 & 1 \\ 1 & 0\end{array}\right), \quad \sigma_{2}=\left(\begin{array}{cc}0 & -i \\ i & 0\end{array}\right), \quad \sigma_{3}=\left(\begin{array}{cc}1 & 0 \\ 0 & -1\end{array}\right)$.

The two-point function is given by

$$
\begin{aligned}
\left\langle\psi_{i, s, x}^{-} \psi_{i^{\prime}, s^{\prime}, y}^{+}\right\rangle_{0} & =\frac{\int P(d \psi) \psi_{i, s, x}^{-} \psi_{i^{\prime}, s^{\prime}, y}^{+}}{\int P(d \psi)} \\
& =\delta_{i, i^{\prime}} \delta_{s, s^{\prime}} g_{s}(x, y)
\end{aligned}
$$

with

$$
g_{i, s}(x, y)=\frac{1}{L^{4}} \sum_{k} e^{i k(x-y)} \frac{\chi_{N}(k)}{-i \sigma_{\mu}^{s} k_{\mu}}
$$

The $n$-point function $\left\langle\psi_{l_{1}, s_{1}, x_{1}}^{\varepsilon_{1}} \ldots \psi_{l_{n}, s_{n}, x_{n}}^{\varepsilon_{n}}\right\rangle_{0}$ is given by the Wick rule. The cutoff function plays a very important role, as it makes the number of Grassmann variables finite; hence, the Grassmann integral is well defined and, at the end, the limit $L \rightarrow \infty$ can be taken.

The currents relevant for electroweak interaction are the $W$ and $B$ ones

$$
\begin{aligned}
j_{W, \mu, x}^{k} & =\frac{1}{2}\left(j_{W, l, \mu, x}^{k}+j_{W, q, \mu, x}^{k}\right), \\
j_{B, \mu, x} & =\frac{1}{2} \sum_{\substack{i=\nu, e, u, d \\
s=L, R}}\left(Y_{i}^{L} j_{i, \mu, x}^{L}+Y_{i}^{R} j_{i, \mu, x}^{R}\right),
\end{aligned}
$$


where $Y_{i}^{L}$ and $Y_{i}^{R}$ are the hypercharges, $Y_{\nu}^{L}=Y_{e}^{L}, Y_{u}^{L}=Y_{d}^{L}$ and

$$
\begin{aligned}
j_{i, \mu, x}^{s} & =\psi_{i, s, x}^{+} \sigma_{\mu}^{s} \psi_{i, s, x}^{-}, \quad j_{W, \mu l, x}^{k}=\Psi_{l, x}^{+} \tau^{k} \sigma_{\mu}^{L} \Psi_{l, x}^{-}, \\
j_{W, \mu, q, x}^{k} & =\Psi_{q, x}^{+} \tau^{k} \sigma_{\mu}^{L} \Psi_{q, x}^{-}
\end{aligned}
$$

with $\tau^{k}$ Pauli matrices. If $\psi$ are classical fields verifying the Dirac equation, the currents $j_{i, \mu, x}^{s}, j_{W, \mu, l, x}^{k}$, and $j_{W, \mu, q, x}^{k}$ in Eq. (7) are separately conserved.

The form of the interaction with classical fields is dictated by the requirement of invariance with respect to a gauge transformation; one gets that the average of the observable $O$, a monomial in the Grassmann variables, is given by

$$
\langle O\rangle_{W, B}=\frac{\int P(d \psi) e^{\int d x\left(g W_{\mu, x}^{k} j_{W, \mu, x}^{k}+g^{\prime} B_{\mu, x} j_{B, \mu, x}\right)} O}{\int P(d \psi) e^{\int d x\left(g W_{\mu, x}^{k} j_{W, \mu, x}^{k}+g^{\prime} B_{\mu, x} j_{B, \mu, x}\right)}} .
$$

Note that such invariance is true at a classical level (that is formally replacing the Grassmann variables with functions) only in the limit of removed cutoff $N \rightarrow \infty$ but is violated at finite $N$. Moreover, even when $N \rightarrow \infty$, the symmetry may be broken at a quantum level in the functional integral (39), which is exactly the anomaly phenomenon.

In order to see this, we can consider the average of the current with respect to Eq. (8). It is computed by expanding in series in the gauge fields; if $A^{0}=B, A^{1}=W$, and the generating function is

$$
e^{\mathcal{W}_{W, B}^{0}}=\int P(d \psi) e^{\int d x\left(g W_{\mu, x}^{k} j_{W, \mu, x}+g^{\prime} B_{\mu, x} j_{B, \mu, x}\right)},
$$

then, if the sum over the choices of $A$ and the combinatorial factor are understood, $g^{0}=g, g^{1}=g^{\prime}$, and $\varepsilon=0,1$,

$$
\begin{aligned}
\left\langle\hat{j}_{B, \mu, p}\right\rangle_{W, B}= & \left.\sum_{n} \frac{1}{n !} \int d p_{1} \cdots d p_{n} \frac{\partial^{n+1} \mathcal{W}_{W, B}^{0}}{\partial B_{\mu, p} \partial A_{\mu_{1}, p_{1}}^{\varepsilon_{1}} \cdots \partial A_{\mu_{n}, p_{n}}^{\varepsilon_{n}}}\right|_{0} \\
& \times g^{\varepsilon_{1}} A_{\mu_{1}, p_{1}}^{\varepsilon_{1}} \cdots g^{\varepsilon_{n}} A_{\mu_{n}, p_{n}}^{\varepsilon_{n}} \delta\left(p+\sum_{i} p_{i}\right)
\end{aligned}
$$

Note that the coefficients are simply the truncated correlations of currents

$\left.\frac{\partial^{n+1} \mathcal{W}_{W, B}^{0}}{\partial B_{\mu, p} \partial A_{\mu_{1}, p_{1}}^{\varepsilon_{1}} \cdots \partial A_{\mu_{n}, p_{n}}^{\varepsilon_{n}}}\right|_{0}=\left\langle\hat{j}_{B, \mu, p} ; \hat{j}_{A^{\varepsilon_{1}, \mu_{1}, p_{1}}} ; \ldots ; \hat{j}_{A^{\varepsilon_{n}}, \mu_{n}, p_{n}}\right\rangle_{0}$

so that the expansion can be represented as the sum of simple Feynman graphs; see Fig. 1. We are, in particular, interested in the conservation of the $U(1)$ currents, which is expressed by $\left\langle\partial_{\mu} j_{B, \mu, x}\right\rangle$.

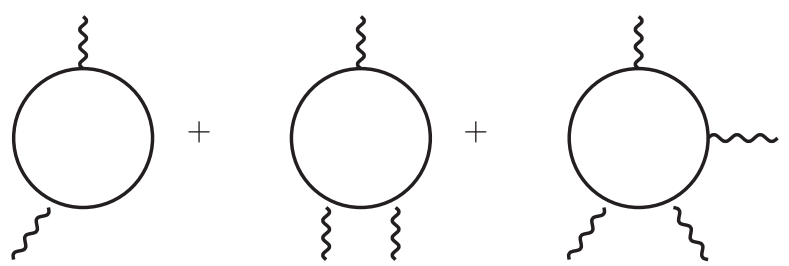

FIG. 1. Graphs contributing to Eq. (10).

\section{B. Ward identities}

We introduce currents $\hat{j}_{\mu, 1, p}=\hat{j}_{\mu, \nu, p}^{L}+\hat{j}_{\mu, e, p}^{L}, \hat{j}_{\mu, 2, p}=$ $\hat{j}_{\mu, u, p}^{L}+j_{\mu, d, p}^{L}$, and $\hat{j}_{p, 3, \mu}=\hat{j}_{\mu, i, p}^{R}$ and a generating function $\mathcal{W}^{0}(A, \phi)$ in which we add to the exponent of Eq. (9) a fermionic source term $\sum_{\alpha=1,2,3} \int d x\left(\psi_{\alpha, x}^{+} \phi_{\alpha, x}^{-}+\psi_{\alpha, x}^{-} \phi_{\alpha, x}^{+}\right)$, where $\quad \psi_{1}=\psi_{L, e}+\psi_{L, \nu}, \quad \psi_{2}=\psi_{L, u}+\psi_{L, d}, \quad$ and $\psi_{3}=\psi_{R}$. Conservation laws are encoded in Ward identities, which can be derived performing in the generating function $\mathcal{W}_{0}(A, \phi)$ the change of variables $i=\nu, e, u, d$ :

$\psi_{i, L, x}^{ \pm} \rightarrow e^{ \pm i \alpha_{L, i, x}} \psi_{i, L, x}^{ \pm}, \quad \psi_{i, R, x}^{ \pm} \rightarrow e^{ \pm i \alpha_{R, i, x}} \psi_{i, R, x}^{ \pm}$,

$\alpha_{L, \nu, x}=\alpha_{L, e, x}, \quad \alpha_{L, u, x}=\alpha_{L, d, x}$

so obtaining, noting that the external currents are invariant,

$e^{\mathcal{W}_{0}(A, \phi)}$

$=\int P(d \psi) e^{-\int d x \psi_{s, i, x}^{+}\left(e^{i \alpha_{s, i, x}} D e^{-i \alpha_{s, i, x}}-D\right) \psi_{s, i, x}^{-}}$

$\times e^{\int d x\left(g W_{\mu, x}^{k} j_{W, \mu, x}+g^{\prime} B_{\mu, x} j_{B, \mu, x}\right)+\int d x\left(\psi_{i, s, x} e^{i i_{i, s, x}} \phi_{i, s, x}+\psi_{i, s, x} e^{-i \alpha_{i, s, x}} \phi_{i, s, x}^{+}\right)}$,

where

$$
D \psi_{s, i, x}=\int d k e^{-i k x} \chi^{-1}(k) \sigma_{\mu}^{s} k_{\mu} \hat{\psi}_{s, i, k}
$$

The fermionic source term acquires a phase but not the current source. The Jacobian of the transformation is unitary as a straightforward consequence of the fact that the number of Grassmann variables $\hat{\psi}_{k}$ is finite; this is an important difference with respect to what happens in (formal) functional integrals with infinitely many variables [5]. The exponent of the fermionic "measure" gets an extra term of the form

$$
\begin{array}{r}
\int d x \psi_{s, i, x}^{+}\left(e^{i \alpha_{s, i x}} D e^{-i \alpha_{s, i, x}}-D\right) \psi_{s, i, x}^{-} \\
=\sum_{i, s} \int d x \delta T_{i, s, x} \alpha_{i, s, x}+O\left(\alpha^{2}\right),
\end{array}
$$

where 


$$
\begin{aligned}
\delta T_{s, i, x}= & \frac{1}{L^{8}} \sum_{k, p} e^{-i p x} \hat{\psi}_{s, i, k}^{+} \sigma_{\mu}^{s}\left(\chi^{-1}(k) k_{\mu}\right. \\
& \left.-\chi^{-1}(k+p)\left(k_{\mu}+p_{\mu}\right)\right) \hat{\psi}_{s, i, k+p}^{-} .
\end{aligned}
$$

Replacing the cutoff function with 1 , one gets that $\chi^{-1}(k) k_{\mu}-$ $\chi^{-1}(k+p)\left(k_{\mu}+p_{\mu}\right)=p_{\mu}$ so that the rhs of Eq. (15) reduces to $\sum_{i, s} \int d x j_{i, s, x} \partial_{\mu} \alpha_{i, s, x}$. Note that the expression $\chi^{-1}$ does not give any problem, as identities have to be understood between correlations. By performing derivatives of Eq. (13) with respect to $\alpha_{s, i, x}$ and to the external fields, we get, see Fig. 2

$$
\begin{aligned}
& p_{\mu}\left\langle\hat{j}_{\mu, \alpha, p} ; \hat{\psi}_{\alpha^{\prime}, k+p}^{-} \hat{\psi}_{\alpha^{\prime}, k}^{+}\right\rangle \\
& =\delta_{\alpha, \alpha^{\prime}}\left(\left\langle\hat{\psi}_{\alpha^{\prime}, k+p}^{-} \hat{\psi}_{\alpha^{\prime}, k+p}^{+}\right\rangle-\left\langle\hat{\psi}_{\alpha^{\prime}, k}^{-} \hat{\psi}_{\alpha^{\prime}, k}^{+}\right\rangle\right)-\left\langle\delta \hat{j}_{\alpha, p} ; \hat{\psi}_{\alpha^{\prime}, k+p}^{+} \hat{\psi}_{\alpha^{\prime}, k}^{+}\right\rangle
\end{aligned}
$$

with $\delta \hat{j}_{1, p}=\delta \hat{j}_{\nu, p}^{L}+\delta \hat{j}_{e, p}^{L}, \delta \hat{j}_{2, p}=\delta \hat{j}_{u, p}^{L}+\delta \hat{j}_{d, p}^{L}, \delta \hat{j}_{3, p}=\delta j_{i, p}^{R}$, and

$$
\begin{aligned}
\delta \hat{j}_{i, p}^{s} & =\frac{1}{L^{4}} \sum_{k} C^{s}(k, p) \hat{\psi}_{s, i, k}^{+} \hat{\psi}_{s, i, k+p}^{-}, \\
C^{s}(k, p) & =\left[\left(\chi^{-1}(k)-1\right) k_{\mu}-\left(\chi^{-1}(k+p)-1\right)\left(k_{\mu}+p_{\mu}\right)\right] \sigma_{\mu}^{s} .
\end{aligned}
$$

The above identity has been written in a form closer to the formal WI writing $\chi^{-1}$ as $\left(\chi^{-1}-1\right)+1$ in Eq. (16). With respect to the formal WI, Eq. (17) has an extra term dependent on the momentum cutoff. The origin of this term can be also understood from the equality between propagators with a momentum cutoff:

$$
\begin{aligned}
g_{s}(k)-g_{s}(k+p)= & g_{s}(k) \sigma_{\mu}^{s} p_{\mu} g(k+p) \\
& +g_{s}(k) C(k, p) g_{s}(k+p),
\end{aligned}
$$

which replaces the identity $g_{s}(k)-g_{s}(k+p)=$ $g_{s}(k) \sigma_{\mu}^{s} p_{\mu} g(k+p)$ in the presence of a cutoff.

We derive now the WI with respect to the currents; by performing derivatives in Eq. (13) with respect to $\alpha$ and $W, B$, we get

$$
\begin{aligned}
& p_{\mu}\left\langle\hat{j}_{\mu, \alpha, p} ; \hat{j}_{A^{\varepsilon_{1}}, \mu_{1}, p_{1}} ; \cdots ; \hat{j}_{A^{\varepsilon_{n}}, \mu_{n}, p_{n}}\right\rangle \\
& \quad=\left\langle\delta \hat{j}_{p, \alpha}, \hat{j}_{A^{\varepsilon_{1}}, \mu_{1}, p_{1}} ; \cdots ; \hat{j}_{A^{\varepsilon_{n}}, \mu_{n}, p_{n}}\right\rangle .
\end{aligned}
$$

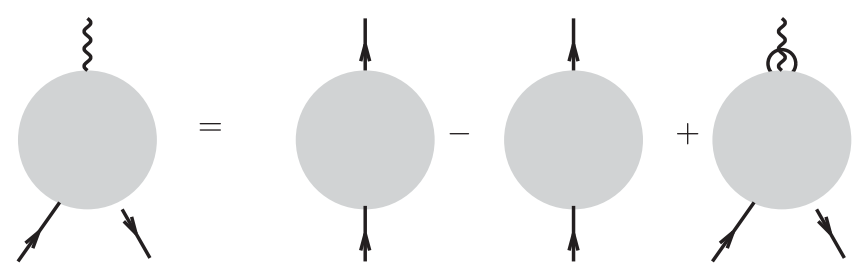

FIG. 2. The WI for the vertex function of Eq. (17); the last term is the vertex involving $\delta j_{\mu}$.
Again we get an extra term with respect to the formal WI proportional to $\delta j_{p, \alpha}$; if such a term would be vanishing, then from Eq. (10) we get the current conservation $p_{\mu}\left\langle\hat{j}_{B, \mu, p}\right\rangle_{W, B}=0$. Even more, the WI (20) with the lhs vanishing is equivalent to the separate conservation of currents of different species. The lhs of Eq. (20) is, however, in general, nonvanishing.

\section{Cancellation condition}

Let us consider now the rhs of Eq. (20) with $n=2$, which is given

$$
\begin{aligned}
& p_{\mu}\left\langle\hat{j}_{s, i, \mu . p} ; \hat{j}_{s, i, \nu, p_{1}} ; \hat{j}_{s, i, \rho, p_{2}}\right\rangle \\
& =\int \frac{d k}{(2 \pi)^{4}} \operatorname{tr} \frac{\chi(k)}{-i \sigma^{s} k_{\mu}} C(k, p) \frac{\chi(k+p)}{-i \sigma_{\mu}^{s}\left(k_{\mu}+p_{\mu}\right)} \\
& \quad \times\left(-i \sigma_{\nu}^{s}\right) \frac{\chi\left(k+p^{2}\right)}{-i \sigma_{\mu}^{s}\left(k_{\mu}+p_{\mu}^{2}\right)}\left(-i \sigma_{\rho}^{s}\right)+\left[\left(\nu, p_{1}\right) \rightarrow\left(\rho, p_{2}\right)\right]
\end{aligned}
$$

and (see [23])

$p_{\mu}\left\langle\hat{j}_{s, p, \mu} ; \hat{j}_{s, p_{1}, \nu} ; \hat{j}_{s, p_{2}, \rho}\right\rangle=\varepsilon_{s} \frac{1}{12 \pi^{2}} \varepsilon_{\nu, \rho, \alpha b} p_{a}^{1} p_{\beta}^{2}+O\left(|\bar{p}|^{3} / \gamma^{N}\right)$,

where $|\bar{p}|=\max \left(\left|p_{1}\right|,\left|p_{2}\right|\right)$ and $\varepsilon_{L}=-\varepsilon_{R}=1$. From Eq. (23) we see that, in addition to terms proportional to the inverse cutoff, there are $N$-independent contributions which are the anomalies in the limit of the removed cutoff.

The average of the $B$ current at second order [see Eq. (10)] is given by

$$
\begin{aligned}
\left\langle\hat{j}_{B, \mu, p} ; \hat{j}_{W, \nu, p_{1}} ; \hat{j}_{W, \mu, \rho, p_{2}}\right\rangle & =\hat{L}_{\mu, \nu, \rho}^{W}\left(p_{1}, p_{2}\right), \\
\left\langle\hat{j}_{B, \mu, p} ; \hat{j}_{B, \nu, p_{1}} ; \hat{j}_{B, \mu, \rho, p_{2}}\right\rangle & =\hat{L}_{\mu, \nu, \rho}^{B}\left(p_{1}, p_{2}\right) .
\end{aligned}
$$

The divergence follows by Eq. (23), and one finds

$$
\begin{aligned}
p_{\mu} \hat{L}_{\mu, \nu, \rho}^{W}\left(p_{1}, p_{2}\right) & =\frac{1}{12 \pi^{2}} \varepsilon_{\nu, \rho, \alpha, \beta} p_{\alpha}^{1} p_{\beta}^{2}\left[\sum_{i} Y_{i}^{L}\right]+R_{\nu, \rho}^{W}\left(p_{1}, p_{2}\right), \\
p_{\mu} \hat{L}_{\mu, \nu, \rho}^{B}\left(p_{1}, p_{2}\right)= & \frac{1}{12 \pi^{2}} \varepsilon_{\nu, \rho, \alpha, \beta} p_{\alpha}^{1} p_{\beta}^{2}\left[\sum_{i}\left(Y_{i}^{L}\right)^{3}-\left(Y_{i}^{R}\right)^{3}\right] \\
& +R_{\nu, \rho}^{B}\left(p_{1}, p_{2}\right)
\end{aligned}
$$

with

$$
\left|R_{\nu, \rho}^{W}\left(p_{1}, p_{2}\right)\right|, \quad\left|R_{\nu, \rho}^{B}\left(p_{1}, p_{2}\right)\right| \leq C \frac{|\bar{p}|^{3}}{\gamma^{N}}
$$

If we require that the current is conserved up to terms to the energy divided by the cutoff scale, we get 


$$
\sum_{i} Y_{i}^{L}=0, \quad \sum_{i}\left(Y_{i}^{L}\right)^{3}-\left(Y_{i}^{R}\right)^{3}=0 .
$$

These are, of course, the same conditions found in the standard electroweak theory with classical gauge fields; indeed, the limit $N \rightarrow \infty$ can be taken safely and exact conservation is found. The condition is verified by elementary particles as $Y_{i}^{L}$ has value $\left(-1,-1, \frac{1}{3}, \frac{1}{3}\right)$ and $Y^{R}$ has value $\left(0,-2, \frac{4}{3},-\frac{2}{3}\right)$ so that one gets $-2+6 \frac{1}{3}=0$ and $6(1 / 3)^{3}+2(-1)^{3}-3(4 / 3)^{3}-3(-2 / 3)^{3}-(-2)^{3}=0$. The conservation of $W$ current does not give further constraint.

We want to investigate if the condition (27) still ensures the current conservation in the interacting case. In such a case, the terms contributing to the divergence of the current are a series of infinitely many Feynman graphs, and a direct verification is impossible. In addition, in order to get nonperturbative results, one needs to keep a finite ultraviolet cutoff, as the Standard Model is not asymptotically free. We ask therefore if also in the interacting case with a finite cutoff the current is conserved up to terms proportional to the energy divided by the cutoff scale provided that the condition (27) is true.

\section{Effective Fermi interaction}

The standard electroweak theory is obtained replacing in Eq. (9) the field $B_{\mu}, W_{\mu}$ with the sum of two fields $B_{\mu}+\tilde{B}_{\mu}$ and $W_{\mu}+\tilde{W}_{\mu}$ (see, e.g., [3]), where $B_{\mu}, W_{\mu}$ are classical background fields and $\tilde{B}, \tilde{W}$ are quantum fields, with a gauge invariant action. The $Z$ and e.m. currents are define $D$ by

$$
\int d x\left(g \tilde{W}_{x, L}^{3} j_{W, \mu}^{3}+g^{\prime} \tilde{B}_{x} j_{B, \mu}\right)=\int d x\left(e A_{\mu, x} j_{\mu, x}^{e m}+\bar{g}^{\prime} Z_{\mu, x} j_{\mu, x}^{Z}\right),
$$

where $\bar{g}^{\prime}=\frac{g}{\cos \theta}, \tanh \theta=g^{\prime} / g, g \sin \theta=g^{\prime} \cos \theta=e$, and the charges are

$$
2 Q_{i}^{s}=I_{3, i}^{s}+Y_{i}^{s}
$$

with $I_{3, i}^{L}= \pm 1$ and $I_{3, i}^{R}=0$ [so that $Q_{i}$ is $(0,-1,2 / 3,-1 / 3)$ ] and

$$
\begin{aligned}
& j_{\mu, x}^{e . m .}=e \sum_{i} Q_{i}\left(j_{i, \mu, x}^{L}+j_{i, \mu, x}^{R}\right), \\
& j_{Z, \mu, x}=\sum_{i, s}\left(I_{3, i}^{s}-\sin ^{2} \theta Q_{i}\right) j_{i, \mu, x}^{s} .
\end{aligned}
$$

From Eq. (29), we see that the proof of charge quantization follows from the quantization of the hypercharges, as $I_{3, i}^{s}$ is quantized.

Because of the Higgs mechanism, the quantum $\tilde{Z}_{\mu}$ and $\tilde{W}$ gauge fields acquire a mass. The effective electroweak theory is obtained integrating the boson fields generating an effective quartic interaction; it is indeed not restrictive to consider only quartic interactions, as monomials of any order in the fields are generated during the RG integrations; see Sec. III. Neglecting for the moment the external gauge fields, the correlations of the effective theory are given by

$$
\langle O\rangle=\frac{\int P(d \psi) e^{V(\psi)} O}{\int P(d \psi) e^{V(\psi)}},
$$

where $P(d \psi)$ is the fermionic integration with renormalized propagator

$$
g_{i, s}(x, y)=\frac{1}{Z_{N, i, s}} \frac{1}{L^{4}} \sum_{k} e^{i k(x-y)} \frac{\chi_{N}(k)}{-i \sigma_{\mu}^{s} k_{\mu}}
$$

and

$$
\begin{aligned}
V(\psi)= & \int d x d y \lambda\left[w_{W}(x, y)\left(j_{W, \mu, x}^{1} j_{W, \mu, y}^{1}+j_{W, \mu, x}^{2} j_{W, \mu, y}^{2}\right)\right. \\
& \left.+w_{Z}(x, y) j_{Z, \mu, x} j_{Z, \mu, y}\right]
\end{aligned}
$$

with $\lambda$ an effective coupling proportional to $g^{2}$ and

$$
\begin{aligned}
& v_{W}(x, y)=\int d k e^{i k(x-y)} \frac{\chi_{N}(k)}{|k|^{2}+M_{W}^{2}}, \\
& v_{Z}(x, y)=A \int d k e^{i k(x-y)} \frac{\chi_{N}(k)}{|k|^{2}+M_{Z}^{2}}
\end{aligned}
$$

with $M=M_{Z}>M_{W}$ and $A$ is a constant to take into account the difference in the effective couplings and masses.

At finite $N$, we can prove that this effective theory has a well-defined nonperturbative meaning, even if in the $L \rightarrow \infty$ limit the functional integrals are infinite dimensional. Indeed, in Sec. III, we prove the following result.

Theorem 1.-The correlations corresponding to Eq. (31) are analytic in $\lambda$ for $|\lambda| \leq\left[\frac{M}{C \gamma^{N}}\right]^{6}$ uniformly as $L \rightarrow \infty$.

Analyticity in the coupling around the origin is a remarkable fact due to the purely fermionic nature of Eq. (B4); indeed, in the presence of bosons, analyticity in zero cannot be true due to the Dyson argument. The estimated radius of convergence is proportional to the gauge mass divided by the cutoff; this reflects the perturbative nonrenormalizability of the theory and implies that the cutoff must be chosen of the order of the gauge mass.

\section{E. Effective electroweak theory and main result}

We include in the effective model the external gauge fields associate to the $B$ and $W$ currents. Because of the interaction, the charges are renormalized, and one needs to introduce bare currents depending on parameters to be fixed so that their values correspond to the physical 
values at low momenta. We introduce therefore the bare background currents

$$
\begin{aligned}
\tilde{j}_{W, \mu, x}^{k} & =\sum_{a=l, q} Z_{N, a, k}^{W} j_{W, a, \mu, x}^{k}, \\
\tilde{j}_{B, \mu, x} & =\sum_{i=\nu, e, u, \frac{d}{s}=L, R} Y_{i}^{s} Z_{N, i, j}^{J} j_{i, \mu, x}^{s}
\end{aligned}
$$

with the parameters $Z_{N, a, k}^{W}$ and $Z_{N, i, s}^{J}$ to be chosen in order to fix the dressed parameters, which can be obtained by the correlations. It is indeed an outcome of our RG analysis in Sec. III that in the analyticity domain $|\lambda| \leq\left[\frac{M}{C \gamma^{N}}\right]^{6}$ the twopoint function is

$$
\left\langle\hat{\psi}_{k, i, s}^{+} \hat{\psi}_{k, i, s}^{-}\right\rangle=\frac{1}{Z_{-\infty, i, s}} \frac{1}{-i \sigma_{\mu}^{s} k_{\mu}}(1+R(k))
$$

with $Z_{-\infty, i, s}$ a nontrivial analytic function of $\lambda$ representing the wave function renormalization and $|R(k)| \leq C|\lambda||k| \gamma^{-N}$. Similarly the three-point functions are $(k \sim k+p \sim \kappa)$

$$
\begin{aligned}
& \left\langle\tilde{j}_{B, \mu, x} ; \hat{\psi}_{i, s, k}^{+} \hat{\psi}_{i, s, k+p}^{-}\right\rangle \\
& \quad=\frac{1}{\sigma_{\mu}^{s} k_{\mu}} \sigma_{\mu}^{s} \frac{1}{\sigma_{\mu}^{s}\left(k_{\mu}+p_{\mu}\right)}\left[\frac{Y_{i}^{s} Z_{i, s,-\infty}^{J}}{Z_{i, s,-\infty} Z_{i, s,-\infty}}+R(k, k+p)\right]
\end{aligned}
$$

with $|R(k, k+p)| \leq C|\lambda||\kappa| \gamma^{-N}$ from which we see that the dressed hypercharge is $\frac{Y_{i}^{s} Z_{i, s,-\infty}^{J}}{Z_{i, s-\infty}}$. A similar expression is found for $\left\langle\tilde{j}_{W, \mu, x} \hat{\psi}_{\nu, s, k}^{+} \hat{\psi}_{e, s, k+p}^{-}\right\rangle$in which the dominant term is proportional to $\frac{Z_{a,-\infty}^{W}}{Z_{\nu, L,-\infty} Z_{e, L,-\infty}}$.

The bare normalization are chosen in order to ensure the following conditions:

$$
Z_{i, s,-\infty}=1, \quad Z_{i, s,-\infty}^{J}=1, \quad Z_{a,-\infty}^{W}=1 .
$$

The first condition ensures that the wave renormalization in the low-energy limit is the same for all particles, the second that the dressed hypercharge is equal to $Y_{i}^{s}$, and the third that the normalizations in the $W$ currents do not depend on the particle species in the low-energy limit. The nontrivial renormalization of the charges is related to the extra terms with $\delta j$ in the WI for the three-point function (17). Such a WI holds also in the interacting case, as $V$ is invariant under the transformation (12). However, the term depending on $\delta j$, which is proportional to the inverse of the cutoff in the noninteracting case, is $N$ independent up to small corrections and $O(\lambda)$ in the presence of interaction; see [21,22] for a similar phenomenon in the $d=1+1$ case.

The effective electroweak theory replacing Eq. (9) is therefore given by

$$
\langle O\rangle_{W, B}=\frac{\int P(d \psi) e^{V(\psi)+\int d x\left(g W_{\mu, x}^{k} \tilde{j}_{W, \mu, x}^{k}+g^{\prime} B_{\mu, x} \tilde{j}_{B, \mu, x}\right)} O}{\int P(d \psi) e^{V(\psi)+\int d x\left(g W_{\mu, x}^{k} \tilde{j}_{W, \mu, x}+g^{\prime} B_{\mu, x} \tilde{j}_{B, \mu, x}\right)}}
$$

with $V$ given by Eq. (33) and $\tilde{j}_{W, \mu, x}^{k}$ given by Eq. (35) with the normalization condition (37) and (38). The response of the $U(1)$ current in the effective theory is given by

$$
\begin{aligned}
\left\langle\hat{j}_{B, \mu, p}\right\rangle_{W, B}= & \left.\sum_{n} \frac{1}{n !} \int d p_{1} \cdots d p_{n} \frac{\partial^{n+1} \mathcal{W}_{W, B}}{\partial B_{\mu, p} \partial A_{\mu_{1}, p_{1}}^{\varepsilon_{1}} \cdots \partial A_{\mu_{n}, p_{n}}^{\varepsilon_{n}}}\right|_{0} \\
& \times g^{\varepsilon_{1}} A_{\mu_{1}, p_{1}}^{\varepsilon_{1}} \cdots g^{\varepsilon_{n}} A_{\mu_{n}, p_{n}}^{\varepsilon_{n}} \delta\left(\sum_{i} p_{i}\right)
\end{aligned}
$$

with the derivative above given by $\left\langle j_{B, \mu, p} ; j_{A^{\varepsilon_{1}, \mu_{1}, p_{1}}} ; \ldots\right.$; $\left.j_{A^{\varepsilon_{n}, \mu_{n}, p_{n}}}\right\rangle$ and

$$
\begin{aligned}
e^{\mathcal{W}(A)} & =\int P(d \psi) e^{V+\int d x\left(g W_{\mu, x}^{k} \tilde{j}_{W, \mu, x}^{k}+g^{\prime} B_{\mu, x} \tilde{j}_{B, \mu, x}\right)} \\
& \equiv \int P(d \psi) e^{V+\mathcal{B}(A)}
\end{aligned}
$$

There are now radiative corrections (see Fig. 3), which could produce extra conditions in order to impose that the current is conserved. This is, however, excluded by the following result.

Theorem 2.-For $|\lambda| \leq\left[\frac{M}{C \gamma^{N}}\right]^{6}$ and choosing $Z_{i, s, N}, Z_{i, s, N}^{J}$, and $Z_{a, N}^{W}$ as functions of $\lambda$ so that Eq. (38) holds, then the three-point function can be written as

$$
\begin{aligned}
\left\langle\tilde{j}_{B, \mu, x} ; \tilde{j}_{B, \nu, x_{1}} ; \tilde{j}_{B, \rho, x_{2}}\right\rangle & =L_{\mu, \nu, \rho}^{B}\left(x, x_{1}, x_{2}\right)+R_{\mu, \nu, \rho}^{1, B}\left(x, x_{1}, x_{2}\right), \\
\left\langle\tilde{j}_{B, \mu, x} ; \tilde{j}_{W, \nu, x_{1}} ; \tilde{j}_{W, \rho, x_{2}}\right\rangle & =L_{\mu, \nu, \rho}^{W}\left(x, x_{1}, x_{2}\right)+R_{\mu, \nu, \rho}^{1, W}\left(x, x_{1}, x_{2}\right)
\end{aligned}
$$

with $\hat{L}_{\mu, \nu, \rho}^{B}$ and $\hat{L}_{\mu, \nu, \rho}^{W}$ verifying Eq. (25) and

$\left|R_{\mu, \nu, \rho}^{1, B}\left(x, x_{1}, x_{2}\right)\right|, \quad\left|R_{\mu, \nu, \rho}^{1, W}\left(x, x_{1}, x_{2}\right)\right| \leq C\left[\frac{1}{\gamma^{N} \delta}\right]^{1 / 2} C_{\delta}$

with $\delta$ the minimal distance between $x, x_{1}$, and $x_{2}$.

We see from Eq. (42) that also in the interacting case the current is conserved up to terms proportional to the inverse of the cutoff scale, provided that the conditions $\sum_{i} Y_{i}^{L}=0$ and $\sum_{i}\left(Y_{i}^{L}\right)^{3}-\left(Y_{i}^{R}\right)^{3}=0$ hold; even if the average of the current is given by a complicate series of graphs, no new

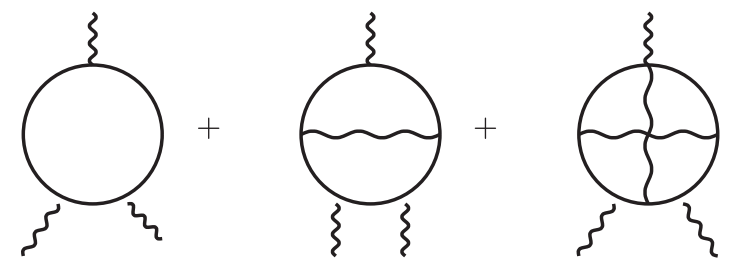

FIG. 3. Graphs contributing to the expansion at $n=2$. 
conditions arises. The crucial bound (43) is nonperturbative; graph expansion is avoided and determinant bounds are used to implement cancellations due to the Pauli principle and ensuring analyticity.

\section{RENORMALIZATION GROUP ANALYSIS}

The proof of the above results is based on exact renormalization group methods, based on the Wilson idea of integrating out high-energy degrees of freedom, obtaining a sequence of effective potentials describing the theory at a fixed energy scale. The starting point is the following decomposition of the cutoff function:

$\chi_{N}(k)=\sum_{h=-\infty}^{N} f_{h}(k), \quad f_{h}(k)=\chi\left(\gamma^{-h} k\right)-\chi\left(\gamma^{-h+1} k\right)$,

so that $f_{h}(k)$ is a smooth cutoff function selecting momenta in $\gamma^{h-1} \leq|k| \leq \gamma^{h+1}$; we also call $\chi_{h}(k)=\sum_{j=-\infty}^{h} f_{j}(k)$ the cutoff function selecting momenta $|k| \leq \gamma^{h}$. We can write the propagator as the sum of two propagators:

$$
g_{i, s}(x, y)=g_{i, s}^{(N)}(x, y)+g_{i, s}^{(\leq N-1)}(x, y)
$$

with $g_{i, s}^{(N)}(x, y)$ is the "single-scale" propagator, describing fermions with momenta $O\left(\gamma^{N}\right)$, while $g_{i, s}^{(\leq N-1)}(x, y)$ is similar to the original propagator with lower ultraviolet cutoff $\gamma^{N-1}$, that is,

$$
\begin{aligned}
g_{i, s}^{(N)}(x, y) & =\frac{1}{Z_{N, i, s}} \frac{1}{L^{4}} \sum_{k} e^{i k(x-y)} \frac{f_{N}(k)}{-i \sigma_{\mu}^{s} k_{\mu}}, \\
g_{i, s}^{(\leq N-1)}(x, y) & =\frac{1}{Z_{N, i, s}} \frac{1}{L^{4}} \sum_{k} e^{i k(x-y)} \frac{\chi_{N-1}(k)}{-i \sigma_{\mu}^{s} k_{\mu}} .
\end{aligned}
$$

Note that the single-scale propagator $g_{i, s}^{(N)}(x, y)$ decays faster than any power for large distances, as a consequence of the fact that the propagator has no singularity in momentum space; instead, $g_{i, s}^{(\leq N-1)}(x, y)$ as a slow power law decay in the coordinate space and a singularity in momentum space.

We use the addition property, saying that the sum of two Gaussian Grassmann variables is a Gaussian Grassmann variable with the propagator given by the sum of propagators. Setting $\mathcal{V}^{(N)}=V+\mathcal{B}$, we can write the generating function (41) as

$$
\begin{aligned}
e^{\mathcal{W}(A)} & =\int P(d \psi) e^{\mathcal{V}^{(N)}(A, \psi)} \\
& =\int P\left(d \psi^{(\leq N-1)}\right) P\left(d \psi^{(N)}\right) e^{\mathcal{V}^{(N)}\left(A, \psi^{(N)}+\psi^{(\leq N-1)}\right)},
\end{aligned}
$$

where $P\left(d \psi^{(N)}\right)$ and $P\left(d \psi^{(\leq N-1)}\right)$ are the Gaussian Grassmann "measures" with propagators $g_{i, s}^{(N)}(x, y)$ and $g_{i, s}^{(\leq N-1)}(x, y)$, respectively. We can now integrate the singlescale field $\psi^{(N)}$, obtaining

$$
\begin{aligned}
\int P\left(d \psi^{(N)}\right) e^{\mathcal{V}^{(N)}\left(A, \psi^{(N)}+\psi^{(\leq N-1)}\right)} & =e^{\sum_{n=0}^{\infty}(1 / n !) \mathcal{E}_{N}^{T}\left(\mathcal{V}^{(N)} ; n\right)} \\
& \equiv e^{\mathcal{V}^{(N-1)}\left(A, \psi^{(\leq N-1)}\right)}
\end{aligned}
$$

where $\mathcal{E}_{n}^{T}(\mathcal{V} ; n)$ are the truncated expectations or cumulants:

$$
\mathcal{E}_{N}^{T}\left(\mathcal{V}^{(N)} ; n\right)=\left.\partial_{\lambda}^{n} \log \int P\left(d \psi^{(N)}\right) e^{\lambda \mathcal{V}^{(N)}}\right|_{\lambda=0},
$$

which can be represented as a sum of connected Feynman graphs with propagator $g^{(N)}$ and external line associated to $\psi^{(\leq N-1)}$. We obtain the following expression:

$\int P(d \psi) e^{\mathcal{V}^{(N)}(A, \psi)}=\int P\left(d \psi^{(\leq N-1)}\right) e^{\mathcal{V}^{(N-1)}\left(A, \psi^{(\leq N-1)}\right)}$

with

$$
\begin{aligned}
& \mathcal{V}^{(N-1)}\left(A, \psi^{(\leq N-1)}\right) \\
& \quad=\sum_{l, m=0}^{\infty} \int d \underline{x} W_{\underline{i}, \underline{s}, l, m}^{(N-1)}(\underline{x}) \prod_{j=1}^{l} \psi_{i_{j}, s_{j}, x_{j}}^{\varepsilon_{j}(\leq N-1)} \prod_{j=1}^{m} A_{\mu_{j}, x_{j}}^{\varepsilon_{j}} .
\end{aligned}
$$

The scaling dimension is $D=4-\frac{3}{2} l-m$, and we can separate the irrelevant terms $D<0$ from the rest. The terms $\psi^{+} \psi^{-}$or $A \psi^{+} \psi^{-}$are generally nonlocal (that is, the fields have different coordinates), and we can split them into a local plus an irrelevant part. In order to obtain this, we define a localization operator $\mathcal{L}$ such that $\mathcal{L}$ gives a vanishing result on the irrelevant terms and

$$
\begin{gathered}
\mathcal{L} \psi_{i, s, x}^{+,(\leq N-1)} \psi_{i^{\prime}, s, y}^{-,(\leq N-1)}=\psi_{i, s, x}^{+,(\leq N-1)} \psi_{i^{\prime}, s, x}^{-,(\leq N-1)} \\
+(x-y)_{\mu} \psi_{i, s, x}^{+,(\leq N-1)} \partial_{\mu} \psi_{i^{\prime}, s, x}^{-,(\leq N-1)}, \\
\mathcal{L} A_{z}^{\varepsilon} \psi_{i, s, x}^{+,(\leq N-1)} \psi_{i^{\prime}, s, y}^{-,(\leq N-1)}= \\
=A_{\mu, z}^{\varepsilon} \psi_{i, s, z}^{+,(\leq N-1)} \psi_{i^{\prime}, s, z}^{-,(\leq N-1)} .
\end{gathered}
$$

By symmetry (see Appendix A), the local part of the quadratic terms can be written as $z_{N-1, i, s} \int d k k_{\mu} \hat{\psi}_{i, s, k}^{+} \sigma_{\mu}^{s} \hat{\psi}_{i, s, k}^{-}$, which can be included in the wave function renormalization:

$$
Z_{N-1, i, s}=Z_{N, i, s}+z_{N-1, i, s} .
$$

In the same way, the contribution from the local part of $A \psi^{+} \psi$ can be included in the current renormalizations, defining 


$$
\begin{aligned}
Z_{N-1, i, s}^{J} & =Z_{N, i, s}^{J}+z_{N-1, i, s}^{J}, \\
Z_{N-1, a, k}^{W} & =Z_{N, a, k}^{W}+z_{N-1, a, k}^{W} .
\end{aligned}
$$

In conclusion, we get

$$
\begin{aligned}
e^{\mathcal{W}(A)} & =\int P(d \psi) e^{\mathcal{V}^{(N)}(A, \psi)} \\
& \left.=\int P\left(d \psi^{(\leq N-1)}\right) e^{\mathcal{L} \mathcal{V}^{(N-1)}\left(A, \psi^{(\leq N-1)}\right)+\mathcal{R} \mathcal{V}^{(N-1)}(A, \psi(\leq N-1)}\right)
\end{aligned}
$$

with propagator

$$
g^{(\leq N-1)}(x, y)=\frac{1}{Z_{N-1, i, s}} \frac{1}{L^{4}} \sum_{k} e^{i k(x-y)} \frac{\chi_{N-1}(k)}{-i \sigma_{\mu}^{s} k_{\mu}},
$$

where

$$
\begin{aligned}
& \mathcal{R V}^{(N-1)}\left(A, \psi^{(\leq N-1)}\right) \\
& =\sum_{l, m}^{*} \int d \underline{x} \tilde{W}_{n, m}^{(N-1)}(\underline{x}) \prod_{j=1}^{l} \partial^{s_{i}} \psi_{i_{j}, s_{j}, x_{j}}^{\varepsilon_{j},(\leq N-1)} \prod_{j=1}^{m} A_{\mu_{j}, x_{j}}^{\varepsilon_{j}},
\end{aligned}
$$

where $\sum^{*}$ has the constraint that if $l=2, m=0$, then $s_{1}+s_{2}=2$ and if $l=2, m=1$, then $s_{1}+s_{2}=1$; that is, the effect of the $\mathcal{R}$ operation is to produce a series with negative scaling dimension. Moreover,

$\tilde{\mathcal{L}} \mathcal{V}^{(N-1)}=\int d x\left(g W_{\mu, x}^{k} \tilde{j}_{W, \mu, x}^{k,(\leq N-1)}+g^{\prime} B_{\mu, x} \tilde{j}_{B, \mu, x}^{(\leq N-1)}\right)$

with

$$
\begin{aligned}
& \tilde{j}_{W, \mu, x}^{k,(\leq N-1)}=\sum_{a=l, q} Z_{N-1, a, k}^{W} j_{W, a, \mu, x}^{k,(\leq N-1)}, \\
& \tilde{j}_{B, \mu, x}^{(1 l e N-1)}=\sum_{\substack{i=\nu, e, \mu, d \\
s=L, R}} Y_{i}^{s} Z_{N-1, i, s}^{J} s_{i, \mu, x}^{s,(\leq N-1)} .
\end{aligned}
$$

The generating function (41) can be exactly written as the rhs of Eq. (55), with a momentum cutoff at a lower scale $\left(\gamma^{N-1}\right.$ instead of $\left.\gamma^{N}\right)$, with modified wave function and charge renormalizations, given by Eqs. (53) and (54); moreover, the interaction is not simply quartic in the fields, as it was in Eq. (41), but is given, according to Eq. (57), by the sum over monomials of any degree with negative scaling dimension. The kernels appearing in the effective potentials and the renormalizations are finite and analytic in $\lambda$. They are indeed expressed by sum Feynman graphs which are finite in the infinite volume limit, as the single-scale propagators are exponentially decreasing; this would be, of course, not true for the Feynman graphs for $\mathcal{W}(A)$, whose propagators has a power law decay. The boundedness of Feynman graphs is, however, not sufficient by itself to get nonperturbative results, as their huge number produces apparently a factorial growth with the order. In the case of fermions, however, cancellations between graphs due to anticommutativity imply convergence of the series; see Appendix B.

The RG analysis continues integrating out the fields $\psi^{(N-1)} \ldots \psi^{(h+1)}$ in a similar way, obtaining

$$
e^{W(A)}=\int P\left(d \psi^{(\leq h)}\right) e^{\mathcal{L} \mathcal{V}^{h}+\mathcal{R} \mathcal{V}^{h}},
$$

where $P\left(d \psi^{(\leq h)}\right)$ is a Grassmann integration with propagator

$$
g_{i, s}^{(\leq h)}=\frac{1}{Z_{i, s, h}} \frac{\chi_{h}(k)}{-i \sigma_{\mu}^{i} k_{\mu}}
$$

and $\mathcal{L} \mathcal{V}^{h}$ and $\mathcal{R} \mathcal{V}^{h}$ are similar with $N-1$ replaced by $h$. Of course, the radius of convergence could decrease as $-h$ increases, but this is not the case, as proved in Appendix B, and one gets that the kernels $W_{n, m}^{(h)}$ are expressed by convergent series in $\lambda$ :

$$
W_{l, m}^{h}=\sum_{n=1}^{\infty} K_{n, l, m}^{h} \lambda^{n}
$$

and

$$
\left|K_{n, l, m}^{h}\right| \leq C^{l+n+m} \gamma^{(4-(3 / 2) l-m) h} \gamma^{\delta_{n}(h-N)}\left[\frac{\gamma^{6 N}}{M^{6}}\right]^{n}
$$

with $\delta_{0}=0$ and $\delta_{n}=\theta=1 / 2$ for $n \neq 0$. The factor $\gamma^{\theta(h-N)}$ is a gain with respect to the "dimensional bound" in the term with at least a $\lambda$ and is due to the dimensional irrelevance of the quartic terms; such an extra factor plays a crucial role in the following. Note that the estimated convergence radius is proportional to the cutoff and mass ratio, as a consequence of the perturbative nonrenormalizability of the theory.

The effective renormalizations verify recursive equations, if $\mathcal{Z}_{h}=\left(Z_{i, s, h}, Z_{i, s, h}^{J}, Z_{a, h}^{W}\right)$ :

$$
\begin{aligned}
\mathcal{Z}_{h-1} & =\mathcal{Z}_{h}+\beta_{\mathcal{Z}}^{h}\left(\lambda ; \mathcal{Z}_{h}, \ldots, \mathcal{Z}_{N}\right), \\
\left|\beta_{\mathcal{Z}}^{h}\right| & \leq \gamma^{\theta(h-N)} C\left[\frac{\gamma^{6 N}}{M^{6}} \lambda\right]
\end{aligned}
$$

where the rhs has an extra factor $\gamma^{\theta(h-N)}$ by Eq. (63), noting that there is no contribution to the $\beta$ function of zero-th order in $\lambda$. The renormalizations are therefore finite:

$$
\mathcal{Z}_{h-1}=\mathcal{Z}_{N}+\sum_{k=h}^{N} \beta_{\mathcal{Z}}^{k}\left(\lambda ; \mathcal{Z}_{k}, \ldots, \mathcal{Z}_{N}\right)
$$


We impose the renormalization conditions; we can look to Eq. (65) as a self-consistence equation, and by contraction methods we find $\mathcal{Z}_{i, s, N}$ as a function of $\lambda$ so that

$$
\mathcal{Z}_{h}=1+O\left(\gamma^{\theta(h-N)} \lambda \frac{\gamma^{6 N}}{M^{6}}\right)
$$

Analyticity stated in Theorem 1 is an immediate consequence of Eq. (63). Note that the denominator of the correlations (the partition function) at finite $L$ is analytic for any $\lambda$ in the whole complex plane, as it is a finitedimensional Grassmann integral; on the other hand, the RG analysis above provides an expansion which coincides order by order and is analytic in a finite domain, so that it fully reconstructs the partition function. The correlation is also analytic, as the denominator is nonvanishing in a finite disk for small $\lambda$ for any $L$ and the numerator is a finitedimensional integral; moreover, it coincides order by order with the expansion found by analyzing the generating function by RG which is also analytic in the same domain so that they coincide and analyticity as $L \rightarrow \infty$ follows.

We use now the above RG analysis for the computation of the three-point function appearing in Eq. (42):

$$
S^{3}\left(x, x_{1}, x_{2}\right)=\left\langle\tilde{j}_{B, \mu, x} ; \tilde{j}_{A^{\varepsilon_{1}}, \mu_{1}, x_{1}} ; \tilde{j}_{A^{\varepsilon_{2}, \mu_{2}, x_{2}}}\right\rangle .
$$

We perform the derivatives of $\mathcal{W}(A)$ given by Eq. (41) with respect to $B_{\mu, x}, A_{\mu_{1}, x_{1}}^{\varepsilon_{1}}$, and $A_{\mu_{2}, x_{2}}^{\varepsilon_{2}}$, and we get

$$
S^{3}\left(x, x_{1}, x_{2}\right)=S_{a}^{3}\left(x, x_{1}, x_{2}\right)+S_{b}^{3}\left(x, x_{1}, x_{2}\right),
$$

where $S_{b}^{3}\left(x, x_{1}, x_{2}\right)$ is obtained by contracting terms with at least a $\lambda$ vertex (see Fig. 4 ) and $S_{a}^{3}\left(x, x_{1}, x_{2}\right)$ is expressed by a renormalized triangle graph of the form, in the case of three $B$ currents,

$$
\begin{aligned}
& \sum_{h_{1}, h_{2}, h_{3}}\left[\prod_{j=1}^{3} \frac{Z_{h_{j}, s, i}^{J}}{Z_{h_{i}, s, i}}\right] \int \frac{d k}{(2 \pi)^{4}} \operatorname{Tr} \frac{f^{h_{1}}(k)}{-i \sigma_{\mu}^{s} k_{\mu}}\left(-i \sigma_{\mu}^{s}\right) \frac{f^{h_{2}}(k+p)}{-i \sigma_{\mu}^{s}\left(k_{\mu}+p_{\mu}\right)} \\
& \times\left(-i \sigma_{\nu}^{s}\right) \frac{f^{h_{3}}\left(k+p^{2}\right)}{-i \sigma_{\mu}^{s}\left(k_{\mu}+p_{\mu}^{2}\right)}\left(-i \sigma_{\rho}^{s}\right)+\left[\left(\nu, p_{1}\right) \rightarrow\left(\sigma, p_{2}\right)\right], \quad
\end{aligned}
$$

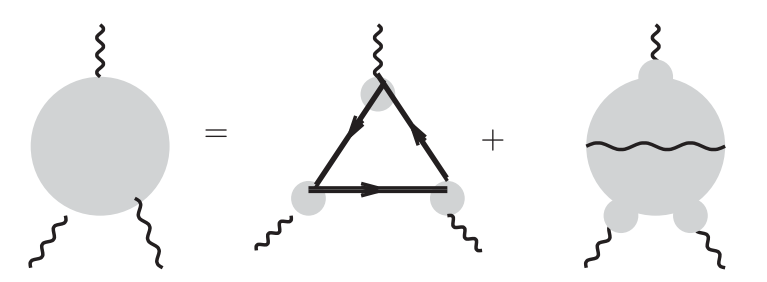

FIG. 4. Graphical representation to $S_{3}$. The first term in the rhs represents $S_{a}^{3}$ and is a sum of triangle graphs; the dots represent the renormalization $Z_{h}^{W}$ or $Z_{h}^{J}$. The other term represents $S_{b}^{3}$, which is the sum of terms with at least a $\lambda$ vertex.

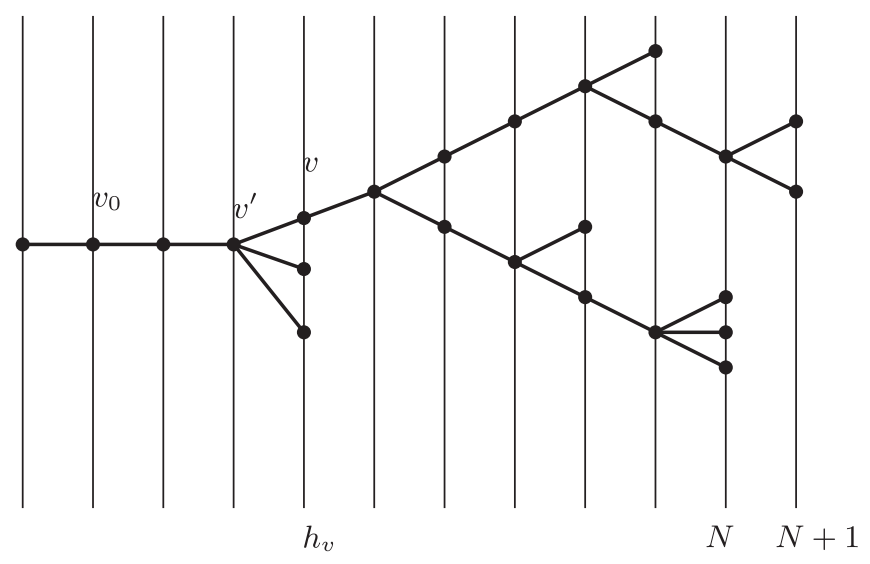

FIG. 5. A labeled tree.

and a similar expression holds for the $B W W$ currents. The main difference with respect to the triangle graphs seen in the noninteracting case is that the wave and the vertex are nontrivial functions of the momentum scale. We can use now Eq. (66) to further decompose the triangle graph as a sum of two terms, one in which $Z_{s, i, h}, Z_{s, i, h}^{J}$, and $Z_{a, h}^{W}$ are replaced by 1 and an extra term. The term in which 1 appears coincides with the noninteracting case using that $\sum_{h=-\infty}^{N} f^{h}=\chi_{N}$. We have now to consider the contribution of $S_{b}^{3}$ and the contribution of the triangle graph with the difference $\mathcal{Z}_{h}-1$. In both cases, there is an extra factor $\gamma^{\theta(h-N)}$; in one case it is due to Eq. (66) and in the other due to factor $\gamma^{\delta_{n}(h-N)}$ in Eq. (63) with $\delta_{n}=\theta$, as it contains a contribution from a $\lambda$ vertex. This factor, as proved in Appendix C, produces the bound (43).

\section{CONCLUSIONS}

We have considered a two-species Standard Model with chiral fermions and a momentum cutoff, with the gauge fields integrated out to produce a current-current interaction. The functional integrals expressing the theory are well defined at a nonperturbative level and analytic in a finite disk in the infinite volume limit. The requirement that the current is conserved up to terms smaller than the inverse of the cutoff, which is the natural condition as gauge invariance is only emerging, still produces the anomaly cancellation condition, implying quantization of charges.

The validity of the analysis holds for a momentum cutoff of the order of the mass of the gauge fields, where fermionic renormalized series are convergent. It would, of course, be interesting to get similar results for larger values of the cutoff; this could be obtained by avoiding integrating out the bosons and considering the standard model electroweak theory with a momentum cutoff. Going beyond perturbation theory is in this case much more difficult, as perturbation theory is expected to be nonconvergent and cluster expansions methods are needed. 
Another interesting question is considering a lattice regularization such that chiral symmetry is nonbroken at a classical level. In the case of QED, the perfect validity of the anomaly nonrenormalization has been recently rigorously established $[23,24]$ with a finite lattice (with emerging Lorentz symmetry), using the lattice regularization in Ref. [25], and it would be interesting to extend such a result, if possible, to chiral theories to get an exact current conservation under the anomaly matching conditions without corrections.

\section{ACKNOWLEDGMENTS}

This work has been supported by MUR (Ministero dell'Universita' e Della Ricerca), PRIN (Progetto Rilevante Interesse Nazionale) 2017, project MaQuMA (Mathematical Quantum Matter), cod. 2017ASFLJR

\section{APPENDIX A: SYMMETRIES}

We prove some symmetry properties of the effective potential (57). In momentum space, the localization operation can be written as

$$
\begin{aligned}
& \mathcal{L} \int d k \hat{W}_{2,0}(k) \hat{\psi}_{i, s, k}^{+,(\leq N-1)} \hat{\psi}_{i^{\prime}, s, k}^{-,(\leq N-1)} \\
& \quad=\int d k\left(\hat{W}_{2,0}(0)+k_{\mu} \partial_{\mu} \hat{W}_{2,0}(0)\right) \psi_{i, s, k}^{+,(\leq N-1)} \psi_{i^{\prime}, s, k}^{-,(\leq N-1)}, \\
& \mathcal{L} \int d k d p \hat{W}_{2,1, \mu}(k, p) A_{\mu, p}^{\varepsilon} \hat{\psi}_{i, s, k}^{+,(\leq N-1)} \hat{\psi}_{i^{\prime}, s, k+p}^{-,(\leq N-1)} \\
& \quad=\int d k d p \hat{W}_{2,1, \mu}(0,0) A_{\mu, p}^{\varepsilon} \hat{\psi}_{i, s, k}^{+,(\leq N-1)} \hat{\psi}_{i^{\prime}, s, k+p}^{-,(\leq N-1)} .
\end{aligned}
$$

The fields $\psi$ have the same chirality, as the propagators are diagonal in the chiral index and the currents have the same chirality. By parity of the propagator, $\widehat{W}_{2,0}(0)=0$. Lorentz symmetry, valid also in the presence of the cutoff, implies that $\partial_{\mu} \widehat{W}_{2,0}(0)$ and $\widehat{W}_{2,1, \mu}(0,0)$ are proportional to $\sigma_{\mu}^{s}$. There are no contributions $\psi_{i}^{+} \partial \psi_{j}^{-}$with $i \neq j$. Indeed, if $i$ and $j$ belong to different families, then such a term would violate the invariance under a global phase transformation $\Psi_{a}^{ \pm} \rightarrow e^{ \pm i \alpha_{a}} \Psi_{a}^{ \pm}$with $a=l, q$. If $i$ and $j$ belong to the same family, then if the field $i$ has $s=R$, it is impossible by a similar argument; if $s=L$, we call $n_{1}$ the number of vertices containing only one field $i$ (say, $e$ ) and $n_{2}$ or $n_{4}$ the number of vertices containing two or four fields, respectively; then $\left(n_{1}-1+2 n_{2}+4 n_{4}\right) / 2$ must be integer; hence, $n_{1}$ is odd but then there is an odd number of fields of the other family $u$ or $d$, and this is impossible. The marginal quadratic terms have therefore the form $z_{N-1, i, s} \int d k k_{\mu} \psi_{i, s, k}^{+} \sigma_{\mu}^{s} \psi_{i, s, k}^{+}$, which can be included in the wave function renormalization. In the same way, there are no contributions to $W_{2,1, \mu}$ with fields with different $i$ index if the source is diagonal in the index, and the nonvanishing terms can be included in the current renormalizations.

\section{APPENDIX B: CONVERGENCE AND ANALYTICITY}

We prove the bound (63). The kernels of the effective potential generated in the renormalization group analysis can be conveniently written as a sum of trees (see Fig. 5), defined in the following way; see, e.g., [19].

Let us consider the family of all trees which can be constructed by joining a point $r$, the root, with an ordered set of $n \geq 1$ points, the end points of the unlabeled tree, so that $r$ is not a branching point. $n$ will be called the order of the unlabeled tree, and the branching points will be called the nontrivial vertices. The unlabeled trees are partially ordered from the root to the end points in the natural way; we shall use the symbol $<$ to denote the partial order. The number of unlabeled trees is $4^{n}$. The set of labeled trees $\mathcal{T}_{h, n}$ is defined associating a label $h \leq N-1$ with the root; moreover, we introduce a family of vertical lines, labeled by an integer taking values in $[h, N+1]$ intersecting all the nontrivial vertices, the end points, and other points called trivial vertices. The set of the vertices $v$ of $\tau$ will be the union of the end points, the trivial vertices, and the nontrivial vertices. The scale label is $h_{v}$ and, if $v_{1}$ and $v_{2}$ are two vertices and $v_{1}<v_{2}$, then $h_{v_{1}}<h_{v_{2}}$. Moreover, there is only one vertex immediately following the root, which will be denoted $v_{0}$ and cannot be an end point; its scale is $h+1$. The end points are associated with $V\left(\psi^{(\leq N)}\right)$, and in such a case the scale is $N+1$ and are named as $\lambda$ or normal end points, or $A$ or special end points $\mathcal{B}\left(\psi^{(\leq N)}, A\right)$ or $\mathcal{L} \mathcal{V}^{h_{v}-1}\left(\psi^{\left(\leq h_{v}-1\right)}, A\right)$. In the case of special endpoints, the scale is $h_{v} \leq N+1$, and there is the constraint that $h_{v}=h_{v^{\prime}}+1$, if $v^{\prime}$ is the first nontrivial vertex immediately preceding $v$; in such a case, they are called special end points.

The effective potential can be written as

$$
\mathcal{V}^{(h)}\left(\psi^{(\leq h)}, A\right)=\sum_{n=1}^{\infty} \sum_{\tau \in \mathcal{T}_{h, n}} \mathcal{V}^{(h)}(\tau),
$$

where, if $v_{0}$ is the first vertex of $\tau$ and $\tau_{1}, \ldots, \tau_{s}\left(s=s_{v_{0}}\right)$ are the subtrees of $\tau$ with root $v_{0}, \mathcal{V}^{(h)}$ is defined inductively by the relation $h \leq N-1$ :

$$
\mathcal{V}^{(h)}(\tau)=\frac{(-1)^{s+1}}{s !} \mathcal{E}_{h+1}^{T}\left[\overline{\mathcal{V}}^{(h+1)}\left(\tau_{1}\right) ; \ldots ; \overline{\mathcal{V}}^{(h+1)}\left(\tau_{s}\right)\right],
$$

where $\mathcal{E}_{h+1}^{T}$ is the truncated expectation and $\overline{\mathcal{V}}^{(h+1)}(\tau)=$ $\mathcal{R} \mathcal{V}^{(h+1)}(\tau)$ if the subtree $\tau_{i}$ contains more then one end point, while if $\tau_{i}$ contains only one end point, $\overline{\mathcal{V}}^{(h+1)}(\tau)$ is $V\left(\psi^{(\leq N)}\right)$ if it is a normal end point (and in such case $h=N-1)$ or if it is a special end point 
$\mathcal{L} \mathcal{V}^{h+1}\left(A, \psi^{(\leq h+1)}\right), h<N-1$, or $\mathcal{B}\left(\psi^{(\leq N)}, A\right)$. We define $P_{v}$ as the set of field labels of $v$ representing the external fields, and if $v_{1}, \ldots, v_{s_{v}}$ are the $s_{v}$ vertices immediately following $v$, then we denote by $Q_{v_{i}}$ the intersection of $P_{v}$ and $P_{v_{i}}$; this definition implies that $P_{v}=\cup_{i} Q_{v_{i}}$. The union of the subsets $P_{v_{i}} \backslash Q_{v_{i}}$ are the internal fields of $v$. Therefore, if $\mathbf{P}_{\tau}$ is the family of all such choices and $\mathbf{P}$ an element, we can write

$$
\begin{aligned}
\mathcal{V}^{(h)}(\tau)= & \sum_{\mathbf{P} \in \mathbf{P}_{\tau}} \int d x_{v_{0}} W_{\tau, \mathbf{P}}^{(h+1)}\left(x_{v_{0}}\right) \\
& \times\left[\prod_{f \in P_{v_{0}}} \psi_{x(f)}^{\varepsilon(f)(\leq h)}\right]\left[\prod_{f} A\left(x_{f}\right)\right],
\end{aligned}
$$

where $W_{\tau, \mathbf{P}}^{\left(h_{v}\right)}\left(x_{v_{0}}\right)$ is defined inductively by the equation

$$
\begin{aligned}
W_{\tau, \mathbf{P}}^{(h+1)}\left(x_{v}\right)= & \frac{1}{s_{v} !}\left[\prod_{i=1}^{s_{v_{i}}} W_{\tau, \mathbf{P}}^{\left(h_{v}+1\right)}\left(x_{v_{i}}\right)\right] \\
& \times \mathcal{E}_{h_{v}}^{T}\left(\tilde{\psi}^{\left(h_{v}\right)}\left(P_{v_{1}} / Q_{v_{1}}\right) ; \ldots ; \tilde{\psi}^{(h)_{v}}\left(P_{v_{s_{v}}} / Q_{v_{s_{v}}}\right)\right),
\end{aligned}
$$

where $\tilde{\psi}^{(h)}(P)=\prod_{f \in P} \psi_{x(f)}^{(h) \varepsilon(f)}$ and $x_{v}$ are the coordinates associated to the vertex $v$. We use the following wellknown representation of the fermionic truncated expectation, if $P$ is a set of indices:

$$
\begin{aligned}
\mathcal{E}_{h}^{T}\left(\tilde{\psi}^{(h)}\left(P_{1}\right) ; \tilde{\psi}^{(h)}\left(P_{2}\right) ; \ldots ; \tilde{\psi}^{(h)}\left(P_{s}\right)\right) \\
\quad=\sum_{T} \prod_{l \in T} g^{(h)}\left(x_{l}-y_{l}\right) \int d P_{T}(\mathbf{t}) \operatorname{det} G^{h, T}(\mathbf{t}),
\end{aligned}
$$

where $T$ is a set of lines forming an anchored tree graph between the clusters of points $x(f)_{f \in P_{i}}$-that is, $T$ is a set of lines-which becomes a tree graph if one identifies all the points in the same cluster. Moreover, $\mathbf{t}=$ $\left\{t_{i, i^{\prime}} \in[0,1], 1 \leq i, i^{\prime} \leq s\right\}, d P_{T}(\mathbf{t})$ is a probability measure with support on a set of $\mathbf{t}$ such that $t_{i, i^{\prime}}=\mathbf{u}_{i} \cdot \mathbf{u}_{i^{\prime}}$ for some family of vectors $\mathbf{u}_{i} \in \mathbb{R}^{s}$ of unit norm. Finally, $G^{h, T}(\mathbf{t})$ is a $(n-s+1) \times(n-s+1)$ matrix, whose elements are given by $G_{i j, i^{\prime} j^{\prime}}^{h, T}=t_{i, i^{\prime}} g^{(h)}\left(x_{i j}-y_{i^{\prime} j^{\prime}}\right)$.

By inserting the above representation, we can write $W_{\tau, \mathbf{P}}^{(h+1)}=\sum_{\mathbf{T}} W_{\tau, \mathbf{P}, \mathbf{T}}^{(h+1)}$, where $\mathbf{T}$ is the union of all the trees $T$.

The determinants are bounded by the Gram-Hadamard inequality, stating that, if $M$ is a square matrix with elements $M_{i j}$ of the form $M_{i j}=\left\langle A_{i}, B_{j}\right\rangle$, where $A_{i}$ and $B_{j}$ are vectors in a Hilbert space with scalar product $\langle\cdot, \cdot\rangle$, then

$$
|\operatorname{det} M| \leq \prod_{i}\left\|A_{i}\right\| \cdot\left\|B_{i}\right\|
$$

where $\|\cdot\|$ is the norm induced by the scalar product. Let $\mathcal{H}=\mathbb{R}^{s} \otimes \mathcal{H}_{0}$, where $\mathcal{H}_{0}$ is the Hilbert space of complex two-dimensional vectors with scalar product $\langle F, G\rangle=\int d k F_{i}^{*}(k) G_{i}(k)$. It is easy to verify that

$$
\begin{aligned}
G_{i j, i^{\prime} j^{\prime}}^{h_{v}, T_{v}} & =t_{i, i^{\prime}} g^{\left(h_{v}\right)}\left(x_{i j}-y_{i^{\prime} j^{\prime}}\right) \\
& =\left\langle\mathbf{u}_{i} \otimes A_{x\left(f_{i j}^{-}\right)}^{\left(h_{v}\right)}, \mathbf{u}_{i^{\prime}} \otimes B_{x\left(f_{i^{\prime} j^{\prime}}^{\prime}\right.}^{\left(h_{v}\right)}\right\rangle,
\end{aligned}
$$

where $\mathbf{u}_{i} \in \mathbb{R}^{s}, i=1, \ldots, s$, are the vectors such that $t_{i, i^{\prime}}=\mathbf{u}_{i} \cdot \mathbf{u}_{i^{\prime}}$ and $A, B$ suitable functions. The integrals over the coordinate are done integrating over the tree $T$ and the interactions, using that for any $K$

$|v(x)| \leq \frac{\gamma^{4 N}}{M^{2}} \frac{C_{N}}{1+(M|x|)^{K}}, \quad \int d x|v(x)| \leq \frac{\gamma^{4 N}}{M^{6}}$

and

$\left|g^{h}(x)\right| \leq C \gamma^{3 h} e^{-\left(\gamma^{h}|x|\right)^{1 / 2}}, \quad \int d x\left|g^{h}(x)\right| \leq C \gamma^{-h}$.

In conclusion, we get

$$
\begin{aligned}
\int d x_{v_{0}}\left|W_{\tau, \mathbf{P}, T}\left(x_{v_{0}}\right)\right| & \leq L^{4} \prod_{v} \frac{1}{s_{v} !} C^{\sum_{i=1}^{s_{v}}\left|P_{v_{i}}\right|-\left|P_{v}\right|} \gamma^{-4 h_{v}\left(s_{v}-1\right)} \\
& \times \gamma^{3 / 2 h_{v}\left(\sum_{i}\left|P_{v_{i}}\right|-\left|P_{v}\right|\right)}\left[\prod_{v} \gamma^{-z_{v}}\right]\left[\frac{\gamma^{4 N}}{M^{6}}\right]^{n},
\end{aligned}
$$

where $z_{v}=1$ if the external fields are $\psi \psi$ or $A \psi \psi \psi$ and zero otherwise. By using that $\left(h_{v}-h_{v^{\prime}}=1\right)$

$$
\begin{aligned}
& \sum_{v}\left(h_{v}-h\right)\left(s_{v}-1\right) \\
& =\sum_{v}\left(h_{v}-h_{v^{\prime}}\right)\left(m_{v}^{4}+n_{v}^{A}-1\right), \\
& \sum_{v}\left(h_{v}-h\right)\left(\sum_{i}\left|P_{v_{i}}\right|-\left|P_{v}\right|\right) \\
& \quad=\sum_{v}\left(h_{v}-h_{v^{\prime}}\right)\left(4 m_{v}^{4}-\left|P_{v}\right|+2 n_{v}^{A}\right),
\end{aligned}
$$

where $m_{v}^{4}$ is the number of $\lambda$ end points following $v$ and $n_{v}^{A}$ is the number of $A$ end points, we get 


$$
\begin{aligned}
& \int d x_{v_{0}}\left|W_{\tau, \mathbf{P}, T}\left(x_{v_{0}}\right)\right| \\
& \leq L^{4} \gamma^{-h\left[-4+\left(3\left|P_{v_{0}}\right| / 2\right)-2 n+n_{v_{0}}^{A}\right]} \\
& \times \prod_{v \text { not e.p. }}\left\{\frac{1}{s_{v} !} C^{\sum_{i=1}^{s_{v}}\left|P_{v_{i}}\right|-\left|P_{v}\right|} \gamma^{-\left(-4+\left(3\left|P_{v}\right| / 2\right)-2 m_{4, v}+n_{v}^{A}+z_{v}\right)}\right\} \\
& \quad \times\left[\gamma^{-2 N n}\right]\left[\gamma^{N} / M\right]^{6 n}
\end{aligned}
$$

and, finally,

$$
\begin{aligned}
\int d x_{v_{0}}\left|W_{\tau, \mathbf{P}, T}\left(\mathbf{x}_{v_{0}}\right)\right| \leq & L^{4} \gamma^{-h d_{v_{0}}} C^{n}|\lambda|^{n}\left[\prod_{\tilde{v}} \frac{1}{s_{\tilde{v}} !} \gamma^{-d_{\tilde{v}}\left(h_{\tilde{v}}-h_{\tilde{v}^{\prime}}\right)}\right] \\
& \times\left[\prod_{\tilde{v}} \gamma^{\left.-2\left(N-h_{\tilde{v}}\right) \bar{m}_{\tilde{v}}\right)}\right]\left[\gamma^{N} / M\right]^{6 n},
\end{aligned}
$$

where $\tilde{v} \in \tilde{V}$ are the vertices on the tree such that $\sum_{i}\left|P_{v_{i}}\right|-\left|P_{v}\right| \neq 0, \quad \tilde{v}^{\prime}$ is the vertex in $\tilde{V}$ immediately preceding $\tilde{v}$ or the root; $\bar{m}_{v}$ is the number of normal end points following $\tilde{v}$ and not any following vertex $\tilde{v} \in \tilde{V}$; and $d_{v}=-4+\frac{3\left|P_{v}\right|}{2}+n_{v}^{A}+z_{v}$. Finally, the number of addenda in $\sum_{T \in \mathbf{T}}$ is bounded by $\prod_{v} s_{v} ! C \sum_{i=1}^{s_{v}}\left|P_{v_{i}}\right|-\left|P_{v}\right|$. In order to bound the sums over the scale labels and $\mathbf{P}$, we first use the inequality

$$
\prod_{\tilde{v}} \gamma^{-d_{\tilde{v}}\left(h_{\tilde{v}}-h_{\tilde{v}^{\prime}}\right)} \leq\left[\prod_{\tilde{v}} \gamma^{-(1 / 2)\left(h_{\tilde{v}}-h_{\tilde{\nu}^{\prime}}\right)}\right]\left[\prod_{\tilde{v}} \gamma^{-\left(3\left|P_{\tilde{v}}\right| / 4\right)}\right],
$$

where $\tilde{v}$ are the nontrivial vertices and $\tilde{v}^{\prime}$ is the nontrivial vertex immediately preceding $\tilde{v}$ or the root. The factors $\gamma^{-(1 / 2)\left(h_{\hat{v}}-h_{\tilde{v}^{\prime}}\right)}$ in the rhs allow one to bound the sums over the scale labels by $C^{n}$. Finally, if there if there is at least a normal end point, the bound improves by a factor $\gamma^{\theta(h-N)}$ as $\bar{m}_{\tilde{v}} \geq 1$ for some $\tilde{v}$ so that, if

$$
\prod_{\tilde{v}} \gamma^{-d_{\tilde{v}}\left(h_{\tilde{v}}-h_{\tilde{v}^{\prime}}\right)}\left[\prod_{\tilde{v}} \gamma^{\left.-2\left(N-h_{\tilde{v}}\right) \bar{m}_{\tilde{v}}\right)}\right] \leq \gamma^{\theta(h-N)} \prod_{\tilde{v}} \gamma^{-\hat{d}_{\tilde{v}}\left(h_{\bar{v}}-h_{\tilde{\nu}^{\prime}}\right)}
$$

with $\hat{d}_{v}=d_{v}-\theta>0$, the sum over scales can be still done. This completes the proof of Eq. (63).

\section{APPENDIX C: THE THREE-CURRENT CORRELATION}

We have now to bound $S_{b}^{3}\left(x, x_{1}, x_{2}\right)$ (68). We can write $S_{b}^{3}\left(x, x_{1}, x_{2}\right)=\sum_{\tau}^{*} S_{\tau}\left(x, x_{1}, x_{2}\right)$, with $\sum_{\tau}^{*}$ the sum over trees with at least one normal end point. With respect to the bound for the kernels of the effective potential (63) obtained in Appendix B, we have to take into account that there is no contribution from the integrals over the coordinates. Given a tree $\tau$, we can associate a tree $\tau^{*}$, which is the tree obtained by $\tau$ by erasing all the vertices not necessary to connect the special end points; given a nontrivial vertex $v \in \tau^{*}$, we call $x_{v}^{*}$ the coordinates associated to end points in $\tau^{*}$ following $v$, and $\delta_{v}$ is the length of the shortest tree graph connecting the points $x_{v}^{*}$. The number of nontrivial vertices $v \in \tau^{*}$ is $\leq 3$. The lack of integration over the external coordinates gives an extra factor $\prod_{v} \gamma^{4 h_{v}\left(S_{v}^{*}-1\right) h_{v}}$, where $S_{v}^{*}$ is the number of branches in $\tau^{*}$ following $v$ (each integration contribute with a factor

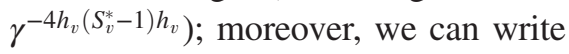

$$
\begin{aligned}
e^{-\sqrt{\gamma^{h}|x|}} & \leq e^{-(1 / 2) \sqrt{\gamma^{h}|x|}} \prod_{k=-\infty}^{0} e^{-c \sqrt{\gamma^{h} \gamma^{k}|x|}} \\
& =e^{-(1 / 2) \sqrt{\gamma^{h}|x|}} \prod_{k=-\infty}^{h} e^{-c \sqrt{\gamma^{k}|x|}}
\end{aligned}
$$

The first factor is used to perform the integrations, and from the second we get a factor $e^{-\left(\gamma^{h_{v}}\left|\delta_{v}\right|\right)^{1 / 2}}$ ] for any nontrivial vertex in $v$ so that, if $m=3$ and $n$ is the number of normal end points $\left(\prod_{\tilde{v}} \gamma^{-\hat{d}_{\tilde{v}}\left(h_{\tilde{v}}-h_{\tilde{\nu}^{\prime}}\right)}=\prod_{v} \gamma^{-\bar{d}_{v}}\right)$,

$$
\begin{aligned}
\left|S_{\tau}\left(x, x_{1}, x_{2}\right)\right| \leq & C^{n}|\lambda|^{n} \gamma^{-h(-4+2 m)} \gamma^{\theta\left(h_{v}^{*}-N\right)}\left[\prod_{v} \gamma^{-\hat{d}_{v}}\right] \\
& \times\left[\prod_{n . t . v \in \tau^{*}} \gamma^{4 h_{v}\left(S_{v}^{*}-1\right) h_{v}} e^{-\left(2^{h_{v}}\left|\delta_{v}\right|\right)^{1 / 2}}\right]
\end{aligned}
$$

with $v_{0}^{*}$ the first nontrivial vertex in $\tau^{*}$. The factor $\gamma^{-h(-4+2 m)}$ apparently forbids one to sum over $h$, and we need to use the decay factors associated to the propagators. In order to do that, we can write

$$
\gamma^{-h(-4+2 m)} \prod_{v_{0} \leq v \leq v_{0}^{*}} \gamma^{-\hat{d}_{v}}=\gamma^{-h_{v_{0}^{*}}(-4+2 m)} \prod_{v_{0} \leq v \leq v_{0}^{*}} \gamma^{-\tilde{d}_{v}}
$$

where $m_{v}=n_{v}^{A}$ for $v_{0} \leq v \leq v_{0}^{*}$ and $\hat{d}_{v}-\left(-4+n_{v}^{A}\right)=$ $\tilde{d}_{v}=3 / 2\left|P_{v}\right|+z_{v}-\theta>0$. We call $S_{v_{0}^{*}}^{1}$ the branches connecting to special $A$ end points. Using that $S_{v_{0}^{*}}=S_{v_{0}^{*}}^{1}+S_{v_{0}^{*}}^{2}$ and $n_{v_{0}^{*}}^{A}=S_{v_{0}^{*}}^{1}+\sum_{i=1}^{S_{v_{0}^{*}}^{2}} n_{v_{i}}^{A}, n_{v_{i}}^{A}$ the number of special end points in $\tau_{i}^{*}$, we have

$$
\begin{aligned}
& -\left(-4+2 n_{v_{0}^{*}}^{A}\right)+4\left(S_{v_{0}^{*}}-1\right) \\
& =-2 S_{v_{0}^{*}}^{1}-\sum_{i=1}^{S_{v_{0}^{*}}^{2}} 2 n_{v_{i}}^{A}+4 S_{v_{0}^{*}}^{1}+4 S_{v_{0}^{*}}^{2} \\
& =2 S_{v_{0}^{*}}^{1}-\sum_{i=1}^{S_{v_{0}^{*}}^{2}}\left(-4+2 n_{v_{i}}^{A}\right) .
\end{aligned}
$$

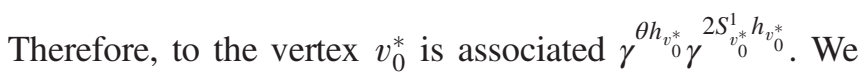
can repeat the same argument on each of the subtrees 
$\tau_{1}^{*}, \ldots, \tau_{S_{0}^{2}}^{*} ;$ moreover, $\gamma^{2 S_{v}^{1} h_{v}} e^{-\left(2^{h_{v}}\left|\delta_{v}\right|\right)^{1 / 2}} \leq C_{\delta}$ as $S_{v}^{1} \leq 3$ so that

$$
\begin{aligned}
& \left|S_{\tau}\left(x, x_{2}, x_{3}\right)\right| \\
& \quad \leq C^{n}|\lambda|^{n} \gamma^{-\theta N}\left[\gamma^{\theta h_{v_{0}^{*}}^{*}} \gamma^{2 S_{v_{0}^{*}}^{1} h_{v_{0}^{*}}} e^{-\left(2^{h_{v_{0}^{*}}^{*}}|\delta|\right)^{1 / 2}}\right]\left[\prod_{v} \gamma^{-\bar{d}_{v}}\right],
\end{aligned}
$$

where $\bar{d}_{v}=\tilde{d}_{v}$ if $v \in \tau^{*}$ and $\bar{d}_{v}=\hat{d}_{v}$ otherwise. The sum over the scale difference is done using that $\bar{d}_{v}>0$; the remaining sum is done using that if $\delta \equiv \gamma^{-h_{\delta}}$, if $1 \leq S^{1} \leq 3$,

$$
\begin{aligned}
& \sum_{h} \gamma^{\left(\theta+2 S^{1}\right) h} e^{-\left(2^{h}|\delta|\right)^{1 / 2}} \\
& \quad=\gamma^{\left(\theta+2 S^{1}\right) h_{\delta}} \sum_{h} \gamma^{\left(\theta+2 S^{1}\right)\left(h-h_{\delta}\right)} e^{-\gamma^{\left(h-h_{\delta}\right) / 2}} \leq C_{\delta}
\end{aligned}
$$

uniformly in $N$. In conclusion, a bound $|\lambda|\left(\frac{1}{\gamma^{N}}\right)^{\theta} C_{\delta}$ is found. A similar bound is found for the corrections coming from the first term, as they have an extra factor $\gamma^{\theta(h-N)}$ from Eq. (66). This concludes the proof of the bound (43).
[1] J. A. Minahan, P. Ramond, and R. C. Warner, Phys. Rev. D 41, 715 (1990).

[2] L. Alvarez-Gaume, An introduction to anomalies, edited by G. Velo and A. S. Wightman, in Fundamental Problems of Gauge Field Theory, NATO ASI Series (Series B: Physics), Vol. 141 (Springer, Boston, 1986).

[3] G. t Hooft, The background field method in gauge field theories, in Functional and Probabilistic Methods in Quantum Field Theory. Proceedings, 12th Winter School of Theoretical Physics, Karpacz (1975), pp. 345-369.

[4] S. L. Adler and W. A. Bardeen, Phys. Rev. 182, 1517 (1969).

[5] K. Fujikawa, Phys. Rev. Lett. 42, 1195 (1979).

[6] C. Kopper and B. Leveque, J. Math. Phys. (N.Y.) 53, 022305 (2012).

[7] S. Adler, in Fifty Years of Yang-Mills Theory, edited by G. 't Hooft (World Scientific, Singapore, 2005).

[8] M. Luscher, Nucl. Phys. B549, 295 (1999).

[9] M. Luscher, Nucl. Phys. B568, 162 (2000).

[10] D. Kadoh and Y. Kikukawa, J. High Energy Phys. 05 (2008) 095.

[11] D. M. Grabowska and D. B. Kaplan, Phys. Rev. Lett. 116, 211602 (2016).
[12] M. Golterman, Nucl. Phys. B, Proc. Suppl. 94, 189 (2001).

[13] J.Preskill, Ann. Phys. (N.Y.) 210, 323 (1991).

[14] J. Soto, Phys. Rev. D 45, 4621 (1992).

[15] S. Coleman and S. L. Glashow, Phys. Rev. D 59, 116008 (1999).

[16] A. M. Polyakov, Gauge Fields and Strings (ITEP, Moscow, 1987).

[17] J. Polchinski, Recent Directions in Particle Theory, in Proceedings of the 1992 Theoretical Advanced Study Institute in Elementary Particle Physics, Boulder, Colorado (World Scientic, 1992), pp. 235-274.

[18] E. Witten, Nat. Phys. 14, 116 (2018).

[19] V. Mastropietro, in Non Perturbative Renormalization (World Scientific, Singapore, 2008), pp. 1-304.

[20] V. Mastropietro, J. Math. Phys. (N.Y.) 48, 022302 (2007).

[21] G. Benfatto, P. Falco, and V. Mastropietro, Commun. Math. Phys. 273, 67 (2007); 285, 713 (2009).

[22] G. Benfatto, P. Falco, and V. Mastropietro, Phys. Rev. Lett. 104, 075701 (2010).

[23] A. Giuliani, V. Mastropietro, and M. Porta, arXiv:1907.00682.

[24] V. Mastropietro, J. High Energy Phys. 03 (2020) 095.

[25] H. B. Nielsen and M. Ninomiya, Phys. Lett. 130B, 389 (1983). 